A C G

publications

\title{
Environmentally Friendly Extraction of Antioxidants from Elettaria cardamomum seeds with Glucose-Citric Acid-Based Natural Deep Eutectic Solvent
}

\author{
Merve Bat Özmatara ${ }^{1^{*}}$ \\ ${ }^{1}$ Department of Chemistry, Gebze Technical University, Gebze- Kocaeli, Türkiye \\ (Received July 26, 2021; Revised August 26, 2021; Accepted September 6, 2021)
}

\begin{abstract}
Elettaria cardamomum belongs to the Zingiberaceae family. It contains components (phenolic compounds, alkaloids, terpenoids) it has antioxidant, anti-inflammatory and antimicrobial effects. The purpose of this study is to research the effect of glucose-citric acid based natural deep eutectic solvent (NADES) on the antioxidant activity of Elettaria cardamomum. In this study, glucose-citric acid was used as a NADES and methanol-water was used as a conventional solvent. The radical scavenging activities, phenolic compound and tannin amounts of the extracts obtained as a result of extraction with two different solvents were found. Antioxidant activities of extracts prepared with natural deep eutectic solvent were found to be higher. It has been shown that NADES, which are less harmful on the health and environment, have low toxicity and are environmentally friendly, can be used in extraction instead of traditional solvents such as hexane, benzene, and methanol.
\end{abstract}

Keywords: Antioxidant activity; Elettaria cardamomum; NADES. (C) 2021 ACG Publications. All rights reserved.

\section{Introduction}

Bioactive compounds such as phenolics, polysaccharides, terpenoids found in plants show antioxidant, antimicrobial, anti-inflammatory and properties [1,2,3]. Depending on their activities, biocompounds derived from different and vegetables herbs have been applied in various formulations in the pharmaceutical, food, cosmetic industry and medicine [4]. The bioactive compounds found in the plant are mostly obtained by extraction processes using aqueous organic solvents such as hexane, methanol, chloroform [5,6]. Generally, the use of traditional solvents such as hexane, benzene, etc. can be detrimental to the surroundings and user health as they show toxicity, volatility, and flammability [7].

Nowadays, environmentally friendly processes are becoming increasingly important as there is a strong concern for reducing energy, water, solvent consumption and carbon emissions [8]. Deep eutectic solvents (DES) have been reported as an alternative to traditional solvents to increase efficiency, improve process selectivity and reduce energy consumption [5]. DES is easily synthesized, less detrimental to the surroundings and less toxic than traditional solvents. These solvents are formed by complexing a hydrogen bond acceptor (HBA) with a hydrogen bond donor (HBD) [9]. When using natural ingredients for DES synthesis, natural deep eutectic solvents (NADES) with lower environmental effects and toxicity are obtained [10].

\footnotetext{
*E-Mail: mervebatt@gmail.com 
Bat Özmatara, Rec. Agric. Food. Chem. (2021) 1:1-2 12-18

Natural deep eutectic solvents (NADES) are bio-based ionic liquids that are composed of two or more compounds such as organic acids, sugars, amines and amino acids. Molecular interactions between NADES components provide inherent properties for these solvents like insignificant vapor pressure, high thermal stability, regulable viscosity, high miscibility [11]. NADES has several advantages such as non-toxicity, low burning, low erosion and low environmental hazards. These properties are crucial to green chemistry. Thus, NADES provides an alternative to avoid problems caused by the use of organic solvents.

Elettaria cardamomum belongs to the Zingiberaceae family. Elettaria cardamomum contains calcium, magnesium, potassium, phosphorus, iron minerals, flavonoids such as catechin, quercetin, carotenoids such as lutein and beta carotene $[12,13,14]$. Elettaria cardamomum shows antioxidant, anticancer, antidiabetic, antifungal, anti-inflammatory properties with its bioactive compounds $[15,16]$.

In the literature, there have been studies in which cardamom is extracted with traditional solvents such as methanol and shows antioxidant activity. However, there is no study on its antioxidant activity when natural deep eutectic solutions are used [17]. The aim of this study is to investigate antioxidant activity using natural deep eutectic solvents (NADES) that can be easily synthesized and are less harmful to the environment and health than traditional solvents.

\section{Materials and Methods}

\subsection{Instrumentation}

The antioxidant activity and phytochemical component amount of Elettaria cardamomum seed extracts were determined using a UV-Vis spectrophotometer (SpectraMax Plus 384 Microplate Reader, California, USA) and Fourier-transform infrared spectroscopy( FT-IR Perkin Elmer 100). The samples were mixed with the aid of a vortex (IKA MS3 from Germany, SIEHE-IKA).

\subsection{Preparation of Glucose-Citric Acid Based NADES}

Citric acid and glucose were weighed at a mole ratio of 1: 1 [18]. Water was added at $15 \%$ by weight to reduce viscosity. In the capped bottle shown in Figure 1, a clear liquid was obtained by stirring continuously for two hours at $80^{\circ} \mathrm{C}$. NADES based on $20 \%$ by weight diluted glucose-citric acid was used in FTIR (Fourier-transform infrared spectroscopy) measurement. Here, glucose-citric acid based natural deep eutectic solvent, abbreviated as G-C NADES.

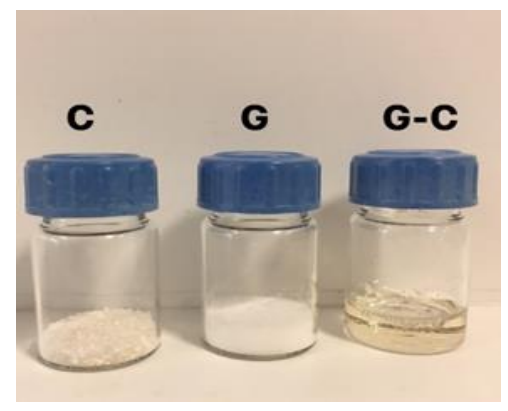

Figure 1. C: Citric acid, G: Glucose, G-C: Glucose-Citric acid based Nades

\subsection{Characterization of Glucose-Citric Acid Based NADES}

FT-IR was used for the characterization of the obtained NADES. The absorbance measures were carried out within the spectra between 600 and $4000 \mathrm{~cm}^{-1}$. 


\subsection{Extraction of Elettaria Cardamomum Seeds}

Elettaria cardamomum seeds were bought from herbalist in Istanbul, Turkey. Cardamomum seeds ( 0.5 grams) were macerated for 24 hours in $5 \mathrm{ml}$ of NADES solvent and $5 \mathrm{ml}$ methanol:water $(1: 1)$. After the incubation period was completed, the seeds were separated by filtering and the obtained extracts were used for the investigation of antioxidant parameters.

\subsection{Determination of Antioxidant Activity}

\subsubsection{DPPH (1,1-diphenyl-2-picrylhydrazyl) Free Radical Scavenging Activity}

The scavenging activity of the DPPH cationic radical is often used to evaluate the antioxidant activity of compounds. The Brand-Williams method was used to test the DPPH radical scavenging activity of the sample [19]. Plant extract $(0.75 \mathrm{ml})$ was mixed with $1.50 \mathrm{~mL}$ of DPPH solution prepared in ethanol $(0.05 \mathrm{mM})$. The mixture was held at room temperature for 30 minutes. At the end of the period, the absorbance at $517 \mathrm{~nm}$ was measured in the UV-Vis spectrophotometer. The scavenging activity of the DPPH radical was calculated by the following equation :

$$
\text { DPPH Scavenging }(\%)=\left(\frac{\text { Acontrol-Asample }}{\text { Acontrol }}\right) \times 100 \quad(\mathrm{Eq} 1)[20]
$$

where Acontrol indicates the absorbance of the control (DPPH solution without sample) and Asample indicates the absorbance of plant sample.

\subsubsection{ABTS [2,2'-azinobis- (3-ethylbenzothiazoline-6-sulfonic acid)] Radical Scavenging Activity}

ABTS radical scavenging activity is based on the reduction of radical and discoloration by the addition of antioxidants to the cationic radical formed by the $\mathrm{K}_{2} \mathrm{~S}_{2} \mathrm{O}_{8}$ oxidation of ABTS [21].

The 7.4 mM ABTS solution and $2.6 \mathrm{mM}$ potassium persulfate solution were mixed in equal volumes and left in the dark at room temperature for 12 hours. $1 \mathrm{~mL}$ of ABTS radical solution was diluted with methanol so that the absorbance at $734 \mathrm{~nm}$ was $1.1 \pm 0.02$. Sample $(150 \mu \mathrm{L})$ and $2850 \mu \mathrm{L}$ of ABTS $^{\cdot+}$ radical solution were mixed and left in the dark for 2 hours. The control solution was prepared using distilled water instead of the sample. At the end of the time, absorbance was measured at $734 \mathrm{~nm}$. In the calculations, ABTS\% radical scavenging effect was found with the formula below [20].

$$
\text { ABTS Scavenging }(\%)=\left(\frac{\text { Acontrol }- \text { Asample }}{\text { Acontrol }}\right) \times 100(\mathrm{Eq} 2)
$$

where Acontrol indicates the absorbance of the control (ABTS solution without sample) and Asample indicates the absorbance of the test sample.

\subsubsection{DMPD (N, N-dimethyl-p-phenylenediamine) Radical Scavenging Activity}

The DMPD radical scavenging method converts the DMPD to the cation radical form (DMPD ${ }^{\circ+}$ ) at acidic $\mathrm{pH}$ or in the presence of an oxidant. $100 \mathrm{~mL}$ of $0.1 \mathrm{M}$ acetate buffer $(\mathrm{pH} 5.3)$ and $0.2 \mathrm{~mL}$ of $0.05 \mathrm{M} \mathrm{FeCl}_{3}$ are added to $1 \mathrm{~mL}$ of $100 \mathrm{mM}$ DMPD solution. $1 \mathrm{ml}$ of this mixture was mixed with 0.5 $\mathrm{ml}$ sample. In the control solution, water was added instead of the sample. After 10 minutes, the absorbance at $505 \mathrm{~nm}$ was measured with a spectrophotometer. DMPD ${ }^{+}$radical scavenging activity was calculated by the following equation [20].

$$
\text { DMPD Scavenging }(\%)=\left(\frac{\text { Acontrol-Asample }}{\text { Acontrol }}\right) \times 100(\mathrm{Eq} 3)
$$


Bat Özmatara, Rec. Agric. Food. Chem. (2021) 1:1-2 12-18

Where Acontrol indicates the absorbance of the control (DMPD solution without sample) and Asample indicates the absorbance of the test sample.

\subsection{Quantitative Phytochemical Analysis}

\subsubsection{Determination of Total Phenolic Compounds}

The method developed by Slinkard-Singleton (1977) was used for total phenolic compound evaluation [22]. After adding $45 \mathrm{~mL}$ distilled water, $1 \mathrm{~mL}$ Folin-Ciocalteau reagent to $1 \mathrm{~mL}$ sample solutions at a $1000 \mu \mathrm{g} / \mathrm{mL}$ concentration, the mixture was thoroughly mixed with a shaking machine. After standing for 3 minutes in this mixture, $3 \mathrm{~mL}$ of $2 \%$ (w/v) $\mathrm{Na}_{2} \mathrm{CO}_{3}$ was added and incubated for 2 hours on the shaking machine. After incubation, the absorbance of the mixtures at $760 \mathrm{~nm}$ was measured. Distilled water was used instead of extract as blank and gallic acid was used as standard. Total phenolic substance content is expressed as the equivalent of the standard used per $\mu \mathrm{g}$ in 1 gram sample.

\subsubsection{Determination of Total Tannin Content}

$0.5 \mathrm{~mL}$ of Folin phenol reagent, $1 \mathrm{~mL}$ of plant extract and $5 \mathrm{~mL}$ of $35 \% \mathrm{Na}_{2} \mathrm{CO}_{3}$ were mixed [23]. The mixture was left at room temperature for 5 minutes. At the end of the period, the absorbance at 640 $\mathrm{nm}$ was read using a UV-Vis spectrophotometer. The tannin content was calculated by the calibration curve of tannic acid and the results are expressed as tannic acid equivalent ( $\mathrm{mg} / \mathrm{g})$.

\section{Results and Discussion}

\subsection{Characterization of Glucose-Citric Acid Based NADES}

The $3650-3200 \mathrm{~cm}^{-1}$ peak is characteristic of hydroxyl groups. The peak at about $1224 \mathrm{~cm}^{-1}$ is the $\mathrm{C}-\mathrm{OH}$ band. The signal at $1025 \mathrm{~cm}^{-1}$ corresponded to the stretching action or rotation of the pyran ring of glucose [24]. In the GC NADES spectrum, frequency shifts and changes in absorbance values were observed compared to single glucose and single citric acid. Peaks in the $\mathrm{C}=\mathrm{O}$ absorbances ranged from 1750 to $1660 \mathrm{~cm}^{-1}$. The results show that hydrogen bonds are well formed between glucose and citric acid in G-C NADES [25].

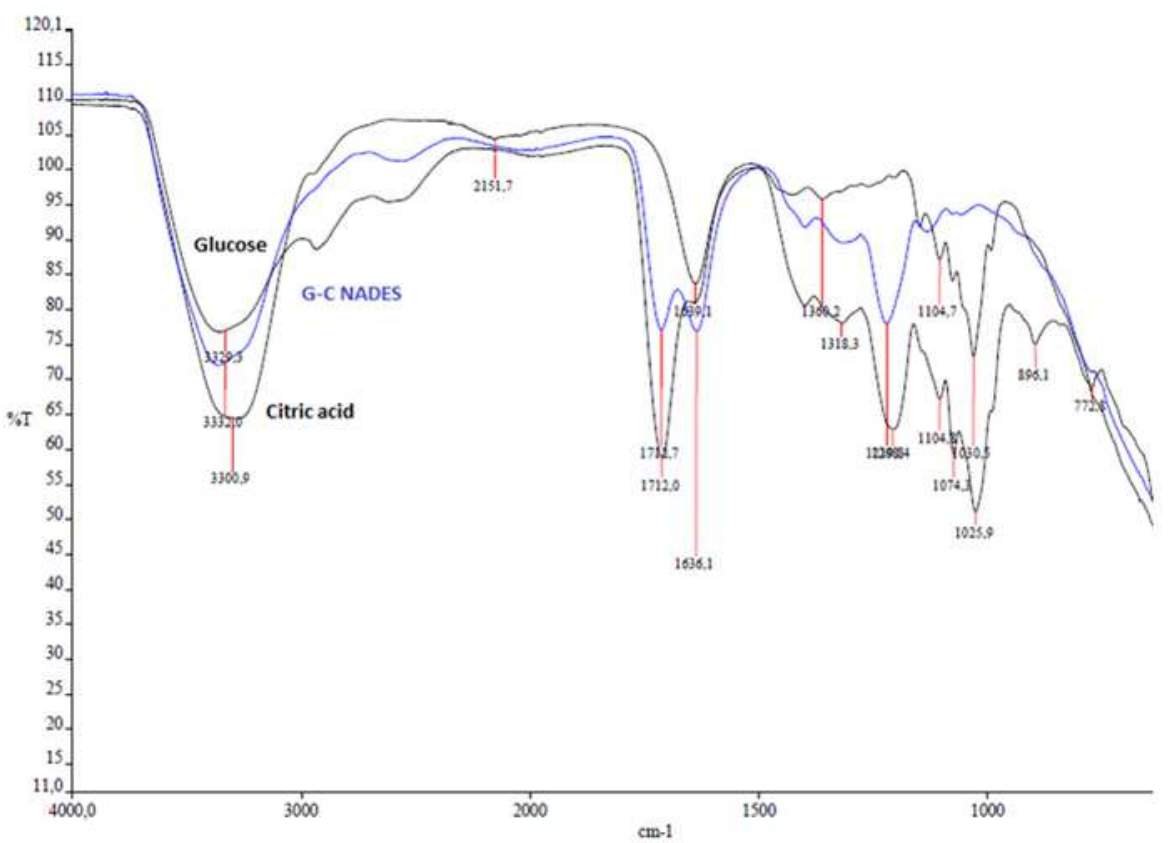

Figure 2. FT-IR of Glucos, citric acid, Glucose-Citric acid-based NADES 


\subsection{Determination of Antioxidant Activity}

Results of the in vitro antioxidant activities using three different assays (DPPH radical scavenging, ABTS radical scavenging, DMPD radical scavenging) were expressed as percent inhibiton. In the DPPH test, the ability of Elettaria cardamomum to act as a donor for hydrogen atoms or electrons to reduce DPPH to DPPH-H was spectrophotometrically measured. In the ABTS test, the reaction between ABTS and potassium persulfate forms the ABTS radical cation $\left(\mathrm{ABTS}^{+}\right)$and a blue green color is observed. When the antioxidant is present, the radical reverts to a colorless state. The DMPD cationic radical $\left(\mathrm{DMPD}^{+}\right)$is formed as a result of a reaction between DMPD and potassium persulfate and is then reduced in the presence of antioxidants. Due to the good extractability of phytochemicals, the antioxidant activity of the extract obtained with Nades solvent was higher than the extraction with methanol:water. The extracts prepared with glucose and citric acid based natural deep eutectic solvent have higher cleaning activity of DPPH, ABTS and DMPD radicals compared to the extract prepared with methanol and water. Results of DPPH, ABTS and DMPD scavenging activities are shown in Fig. 3 .

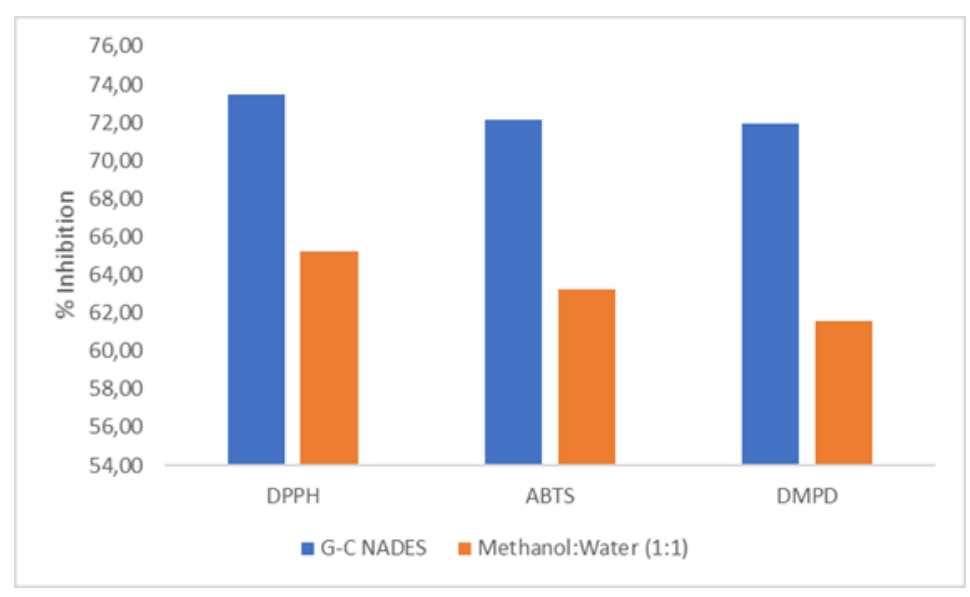

Figure 3. DPPH, ABTS, DMPD radical scavenging activities of extracts

\subsection{Quantitative Phytochemical Analysis}

The phytochemical analysis of the extracts are shown in Table 1. The physical or physicochemical properties of the solvent often affect the extractability of target compounds from a solid matrix. Due to its good extractability, the amount of phytochemicals in the extraction with Nades solvent was found to be higher than the extraction with methanol:water. The level of total phenolic compound was found higher in G-C NADES (198.25 $\mu \mathrm{g}$ gallic acid equivalent (QE)/g) and lower in methanol:water extract $(146.32 \mu \mathrm{g}$ gallic acid equivalent $(\mathrm{GE}) / \mathrm{g})$. When the total tannin content amounts were measured, it was seen that the tannin level of the G-C NADES extract (132.085 mg tallic acid equivalent (TAE)/g extract) was higher than the methanol: water extract ( $82.39 \mathrm{mg} \mathrm{TAE} / \mathrm{g}$ extract). 
Bat Özmatara, Rec. Agric. Food. Chem. (2021) 1:1-2 12-18

Table 1. Quantitative phytochemical analysis of extract

\begin{tabular}{ccc}
\hline Samples & Total Phenolic Content $(\mu \mathrm{g}$ GAE/g) & Total Tannin Content $(\mathrm{mg}$ TAE/g) \\
\hline G-C NADES & 198.25 & 132.085 \\
Methnaol:Water (1:1) extract & 146.32 & 82.39 \\
\hline
\end{tabular}

\section{Conclusion}

A novel, simple, and environmentally friendly NADES method was developed for the extraction of antioxidants from the seeds of Elettaria cardamomum. The amount of phenolic compound and tannin in the extract prepared with NADES and the radical scavenging activity in connection with these were found to be higher than the extract prepared with methanol-water. Finally, it has been observed that the present natural deep eutectic solvent is an effective and suitable medium for the extraction of active biocomponents such as phenolic compound.

\section{ORCID}

Merve Bat Özmatara: 0000-0002-6912-8825

\section{References}

[1] S. Ndomo, F. Djikeng, G. Teboukeu, H. Doungue, H. Foffe, C. Tiwo and H. Womei (2021). Nutritional value, phytochemical content, and antioxidant activity of three phytobiotic plants from West Cameroon, J. Agric. Food Res. 3, 100105.

[2] A. Ashraf, R. Sarfraz, M. Rashid and M. Shahid (2015). Antioxidant, antimicrobial, antitumor, and cytotoxic activities of an important medicinal plant (Euphorbia royleana) from Pakistan, J. Food Drug Anal. 23(1), 109-115.

[3] M. Moreno, I.C. Zampini and M.I. Isla (2020). Antifungal, anti-inflammatory and antioxidant activity of bi-herbal mixtures with medicinal plants from Argentinean highlands, J. Ethnopharmacol. 253, 112642.

[4] C.Y.H. Djande, L.A. Piater, P.A. Steenkamp, M.E. Madala and I.A. Dubery (2018). Differential extraction of phytochemicals from the multipurpose tree, Moringa oleifera, using green extraction solvents, $S$. Afr. J. Bot. 115, 81-89.

[5] C.M.G.C. Renard (2018). Extraction of bioactives from fruit and vegetables: State of the art and perspectives, LWT-Food Sci. Technol. 93, 390-395.

[6] M.G. Bogdanov, I. Svinyarov, R. Keremedchieva and A. Sidjimov (2012). Ionic liquid supported solidliquid extraction of bioactive alkaloids. I. New HPLC method for quantitative determination of glaucine in Glaucium flavum Cr. (Papaveraceae), Sep. Purif. Technol. 97, 221-227.

[7] C. Bakirtzi, K. Triantafyllidou and D.P. Makris (2016). Novel lactic acid-based natural deep eutectic solvents: Efficiency in the ultrasound-assisted extraction of antioxidant polyphenols from common native Greek medicinal plants, J. Appl. Res. Med. Aroma. 3, 120-127.

[8] F. Chemat, N. Rombaut, A. Meullemiestre, M. Turk, S. Perino and M. Abert-Vian (2017). Review of green food processing techniques. Preservation, transformation, and extraction, Innov. Food Sci. Emerg. Technol. 41, 357-377.

[9] A.P. Abbott, D. Boothby, G. Capper, D.V. Davies and R.K. Rasheed (2004). Deep eutectic solvents formed between choline chloride and carboxylic acids: Versatile alternatives to ionic liquids, J. Am. Chem. Soc. 126, 9142-9147.

[10] Y.H. Choi, J. Van Spronsen, Y. Dai, M. Ververne, F. Hollmann, I.W.C.E. Arends, G.J. Witkamp and R. Verpoorte (2011). Are natural deep eutectic solvents the missing link in understanding cellular metabolism and physiology? Plant Physiol. 156, 1701-1705.

[11] L. Benvenutti, A. Camargo, F. Zielinski and R. Ferreira (2020). NADES as potential solvents for anthocyanin and peçtin extraction from Myrciaria cauliflora fruit by-product: In silico and experimental approaches for solvent selection, J. Mol. Liq. 315, 113761.

[12] M.D. Sontakke, H.M. Syed and A.R. Sawate (2018). Studies on extraction of essential oils from spices (Cardamom and Cinnamon), Int. J. Chem. Studies. 6, 2787-2789.

[13] K.I. Ereifej, H. Feng, T.M. Rababah, S.H. Tashtoush, M.H. Al-U'datt, G.J. Al-Rabadi, P. Torley and M. Alkasrawi (2015). Microbial status and nutritional composition of spices used in food preparation, Food Nutr. Sci. 6, 1134-1140. 


\section{Antioxidants from Elettaria cardamomum}

[14] K. Ashokkumar, A. Pandian, M. Murugan, M.K. Dhanya, T. Sathyan, P. Sivakumar, R. Surya and T.D. Warkentin (2019). Profiling bioactive flavonoids and carotenoids in select south Indian spices and nuts, Nat. Prod. Res. 34(9), 1306-1310.

[15] R. Hamzaa and N. Osman (2012). Using of coffee and cardamom mixture to ameliorate oxidative stress induced in $\gamma$-irradiated rats, Biochem. Ana. Biochem. 1, 113-119.

[16] H. Winarsi, N.D. Sasongko, A. Purwanto and I. Nuraeni (2014). Effect of cardamom leaves extract as antidiabetic, weight lost and hypocholesterolemic to alloxan-induced Sprague Dawley diabetic rats, Int. Food Res. J. 21, 2253-2261.

[17] A. Saeed, B. Sultana, F. Anwar, M. Mushtaq, K.M. Alkharfy and A.H. Gilani (2014). Antioxidant and Antimutagenic Potential of Seeds and Pods of Green Cardamom (Elettaria cardamomum), Int. J. Pharmacol. 10(8), 461-469.

[18] H. Vanda, R. Verpoorte, P.G.L. Klinkhamer and Y.H. Choi (2019). Natural deep eutectic solvents: from their discovery to their applications, Deep Eutectic Solvents: Synthesis, Properties and Applications., 6181. Wiley-VCH Verlag GmbH \& Co. KGaA.

[19] W. Brand-Williams, M.E. Cuvelier and C. Berset (1995). Use of a free radical method to evaluate antioxidant activity, $L W T$. 28, 25-30.

[20] O. Cioancă, C. Mircea, L. Hritcu, A. Trifan, M. Mihasan, A.C. Aprotosoaie, S. Robu, E. Gille and M. Hancianu (2015). In vitro-in vivo correlation of the antioxidant capacity of Salviae aetheroleum essential oil, Farmacia, 63(1), 34-39.

[21] M. Arnao, A. Cano, J. Alcolea and M. Acosta (2001). Estimation of free radical-quenching activity of leaf pigment extracts, Phytochem. Anal. 12(2), 138-143.

[22] K. Slinkard, V.L. Singleton (1977). Total phenol analysis: automation and comparison with manual methods, Am. J. Enol. Viticult. 28, 49-55.

[23] S.H. Schanderl (1970). Methods in food analysis, New York: Academic Press, 709 pp.

[24] K. Kajiwara, T. Uemura, H. Kishikawa, K. Nishida, Y. Hashiguchi, M. Uehara, M. Sakakida, K. Ichinose and M. Shichiri (1993). Noninvasive measurement of blood glucose concentrations by analysing fourier transform infra-red absorbance spectra through oral mucosa, Med. Biol. Eng. Comput. 31, 17-22.

[25] R. Xin, S. Qi, C. Zeng, F.I. Khan, B. Yang and Y. Wang (2017). A functional natural deep eutectic solvent based on trehalose: Structural and physicochemical properties, Food Chem. 217, 560-567.

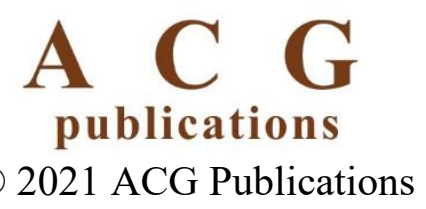

